\title{
Wide-band Relativistic Doppler Effect Visualization
}

\author{
Ping-Kang Hsiung ${ }^{*}$ Robert H. Thibadeau ${ }^{\dagger}$ \\ Christopher B. Cox ${ }^{\ddagger}$ Robert H. P. Dunn ${ }^{\S}$ \\ Michael Wu" Paul Andrew Olbrich" \\ Carnegie Mellon University \\ Pittsburgh, Pennsylvania 15213 \\ "I wonder what the world would look like if I could ride a beam of light."
}

\begin{abstract}
One of the most visible aspects of special relativity is the relativistic Doppler effect - the dependence of observed radiation wavelengths upon the velocity of the source and the viewing conditions.

In this paper, we present a flexible and efficient method to simulate the Doppler shift. This new method has the following features:

- Surface properties and light composition are represented by splines as functions of wavelength. The entire electromagnetic (EM) spectrum can therefore be represented efficiently.

- Doppler shift and shading operations are performed through the manipulation of spline coefficients. The evaluation of the spline functions is carried out at the end of each shading loop to generate the display $R, G, B$ values.
\end{abstract}

This method simplifies the management of the shift and reduces the calculations necessary to maintain a spectral description of lights and surfaces.

The study of astrophysical phenomenon, which are being color-shifted by individual recession velocity and by the expansion of the universe, requires the use of knowledge about the Doppler effect. Our simulations may contribute visual insight and understanding that enhances such knowledge.

*Department of Electrical and Computer Engineering, Camegie Mellon University. (412) 268-2524.pkh@cs.cmu.edu

†Imaging Systems Laboratory, The Robotics Institute, Camegie Mellon University. rht@cs.cmu.edu

¿Department of Physics, Camegie Mellon University.

$\S$ Department of Art, Carnegie Mellon University.

TDepartment of Electrical and Computer Engineering, Camegie Mellon University.

ll Department of Mathematics, Camegie Mellon University.

This research was partially supported by Imaging Systems Laboratory, The Robotics Institute, Camegie Mellon University.

\section{Introduction}

We have developed an algorithm to visualize the 4dimensional spacetime model in special relativity. The algorithm, called REST-frame, builds on the conventional graphical technique of ray-tracing, but encompasses finite light-speed and special relativity in modeling ray propagation. Objects in relativistic motion are shown to undergo spatial distortion, time dilation and Doppler color shift.

Whereas the spatial and temporal effects simulations incur less than $5 \%$ computational overhead over the original ray-tracing algorithm, the Doppler shift simulation is far more expensive. In simulating the Doppler shift, object surface properties and light composition are represented as spectral curves, covering a very large range of wavelengths. These curves must be shifted, multiplied and added with every shading calculation. In our previous attempt these curves were represented by discrete samplings at fixed intervals. Each curve would be re-sampled after a shift, and multiplied and added component-wise for shading. This approach incurred over $100 \%$ overhead in our earlier implementation, and had other limitations.

In this paper, we report a flexible and efficient method to simulate the Doppler color shift. This new method focuses on removing the limitations in the prior investigation, and has the following features:

- The spectral curves of surface properties and light composition are represented by spline functions of wavelength. These functions can cover the entire EM wave bandwidth, and incorporate the thermal radiation of objects into the surface property description. In particular, a temperature-dependent emission spectral distributioncan be assigned to each object for imaging the non-visible thermal spectra which may become visible due to blue shift.

- Doppler shift and shading operations are performed through the manipulation of spline coefficients. The evaluation of the spline functions, which is computationally expensive, is only carried out once - at the 
end of each shading loop for generating the display $R, G, B$ values.

\section{Background}

\subsection{Special relativity and Doppler shift}

Einstein's special theory of relativity (1905) rests on two postulates [7]:

1. Physical laws must be the same for observers in all inertial reference systems. ${ }^{1}$

2. Light speed is a constant as measured in all frames, and is independent of the light source motion relative to the observer.

When a physical event is measured, all frames must record their individually defined space and time measurements. Among the frames, the different measurements about the same physical event are co-related by the Lorentz Transformation.

The Doppler frequency shift is observed when an electromagnetic (EM) radiation source is moving at a speed comparable to that of the EM wave it is emitting. In effect, the observed frequency spectrum of an EM source varies, and depends on the relative velocity of the source motion as well as the angle that the radiation reaches the observer. The dependency is expressed mathematically by equation 1 :

$$
f=\frac{1}{\gamma(1+\vec{e} \cdot \vec{\beta})} f^{\prime}=\alpha f^{\prime}
$$

in which

$f^{\prime}$ : frequency of light in its proper frame $S^{\prime}$.

f : frequency of light observed in observer frame $S$.

$\vec{e}$ : observed (normalized) direction of light in S.

$\vec{\beta}$ : velocity of $S$ ' relative to $S$.

Figure 1 shows the graphs of equation (1) at speeds $0.5 \mathrm{c}$, $0.9 \mathrm{c}$ and $0.99 \mathrm{c}$. The horizontal axis is the viewing angle enumerated from $-90^{\circ}$ to $90^{\circ}$. $0^{\circ}$ means the viewing direction is exactly perpendicular to the direction of object motion. The vertical axis represents the shift factor $(\alpha$ in the equation) of the observed frequency change.

In figure 1, the left side portions of the curves reveals the up-shift of observed frequency ("blue shift") of approaching objects, and the right sides of the curves the down-shift ("red shift") of receding objects. Also, the viewing angle that the shift factor evaluates to 1.0 (the "crossover" angle for color shift) is negative in each curve. Near the

\footnotetext{
${ }^{1}$ Also referred to as inertial frames or, in short, frames. A reference system is inertial if it is nonaccelerating. A frame in which an object is stationary is called the proper frame or rest frame of the object.
}

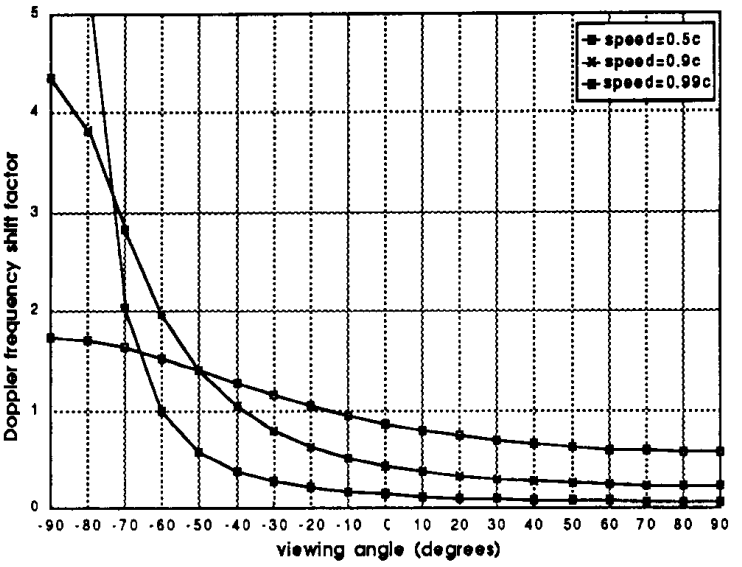

Figure 1: Viewed frequency shift factor versus viewing direction.

crossover angle, an object will be recorded by a camera to exhibit both blue and red shifts simultaneously. More discussions on the curves can be found in [4].

\subsection{Ray-tracing relativity}

Our work on REST-frame relativistic ray-tracing[3][5] has emphasized the incorporation of time in ray modeling finite light-speed and relativity physics are built into the rules of ray propagation and object/camera motion. Rays are "fired" from the camera point at imaging time and travel backward in 4D spacetime to their source events.

\subsection{Operations in Doppler effect simulation}

The Doppler effect can be modeled in relativistic raytracing through the inclusion of frequency shift and modification to shading.

In ray-tracing, each ray makes a contribution to the final color of the pixel. This includes, for each pixel, the primary (screen) ray and recursively generated secondary rays for reflection and refraction. The recursive shading process builds a "shade tree" for each pixel [1], where each node of the tree represents the color of an illuminated surface. Branches are formed starting from the surface and leading to a contributing light source, whether it is an actual light, the background void, or another surface. The final color of the pixel is the weighted sum of the contributing sources at tree nodes. Each node is weighted according to the properties of the surface. This weighted summation - a simplified numerical integration - approximates the true physical phenomena of energy transfer modeled by the integral rendering equation [6]. 


\subsubsection{Doppler operations}

In this context, the Doppler operations of frequency shift and shading are incorporated as follows:

- frequency shift (or wavelength shift): The color of the contributing source returned by each ray is adjusted according to equation 1 , in which $\vec{e}$ is the direction of the ray, and $\vec{\beta}$ is the relative velocity of the contributing source and the illuminated surface. The background is assumed to be stationary relative to the camera.

- shading: This is the weighted summation process. At each node of the shade tree, the shifted color of a contributing source is multiplied by the optical property of the illuminated surface to give the true color of the node.

These operations are illustrated in figure 2 and 3 (the illustration shows the shift of wavelength, in nanometer unit.) Note that a frequency shift of a spectral curve by a con-
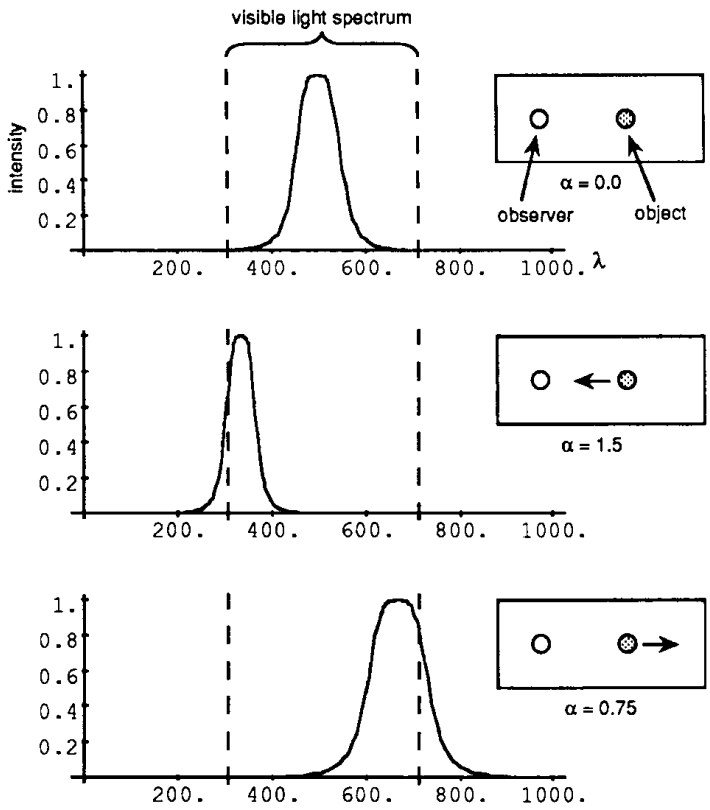

Figure 2: Doppler shift operations (a) source intensity (b) after upshift adjustment (c) after downshift adjustment.

stant factor deforms the curve in addition to moving it. A up-shifted curve thus "spreads out" more than its original; a down-shift "contracts" a curve.

The final spectral curve from each shade tree (corresponding to each screen pixel) can than be converted to RGB for display.

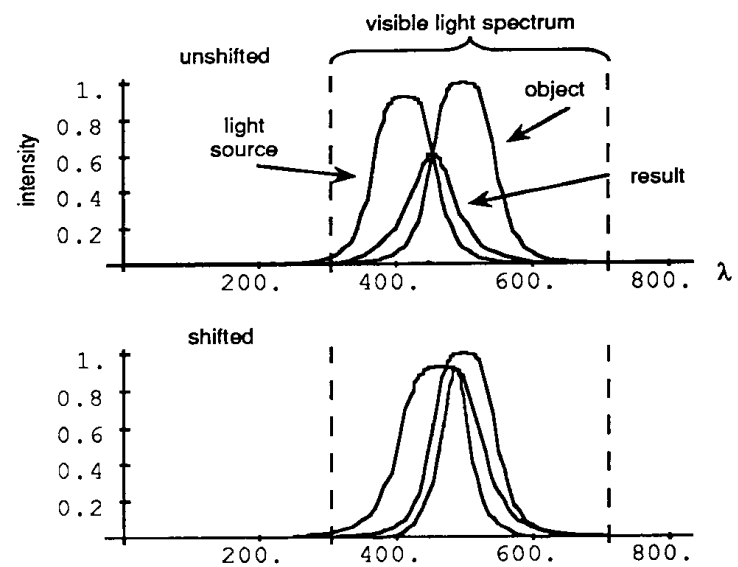

Figure 3: Weighted summation of shifted source (a) unshifted shading (b) shifted shading.

\subsection{Wide band representation requirement}

Doppler shift can be severe due to high speed head-on motion or the cascading of smaller shifts through multiple reflections. Light in the visible range can be shifted out of the visible region, and longer or shorter wavelengths may become visible. To cover (and accurately model) this shift complexity, the spectral curves need to have a very wide-band representation.

An additional motivation for having curves of wide bandwidth range is that for astrophysical applications, we wish to move the "observation window" across the EM spectrum and render images at any selected range (e.g. to compare with images of stars, quasars and galaxies from radio telescope, IR or UV cameras and Gamma- and X-ray detectors).

\subsection{Photon flux consideration}

The square of the shift factor $\alpha$ determines to what extent intensity is enhanced or reduced. Intensity is observed to greatly increase in the direction of motion as a relativistic source moves at larger increments of light speed. As a source moves away from the observer, light intensity is seen to decrease. Isotropic emission from a relativistic source appears to be focused forward much like a headlight beam; an effect also called relativistic beaming [8]. Simulation of this intensity effect quickly caused color saturation, therefore it was used minimally in this imaging context. 


\section{Prior work in visualizing Doppler effect}

In [4], we reported our preliminary implementation of Doppler shift in REST-frame. Object surface properties and light source spectral composition were represented by large data arrays that were the discrete samples of spectral curves. Such representation was expensive to operate on and had other limitations, such as limited numerical accuracy.

\subsection{Shift and shading in spectral line based implementation}

Based on the spectral sample representation, the Doppler operations of shift and shading were performed in the following way:

- frequency shift: The lines were shifted and resampled at fixed frequencies for every shift operation. This included the interpolation of the shifted samples to calculate the new samples.

Figure 4 shows the discrete representation of the shifts in figure 2.
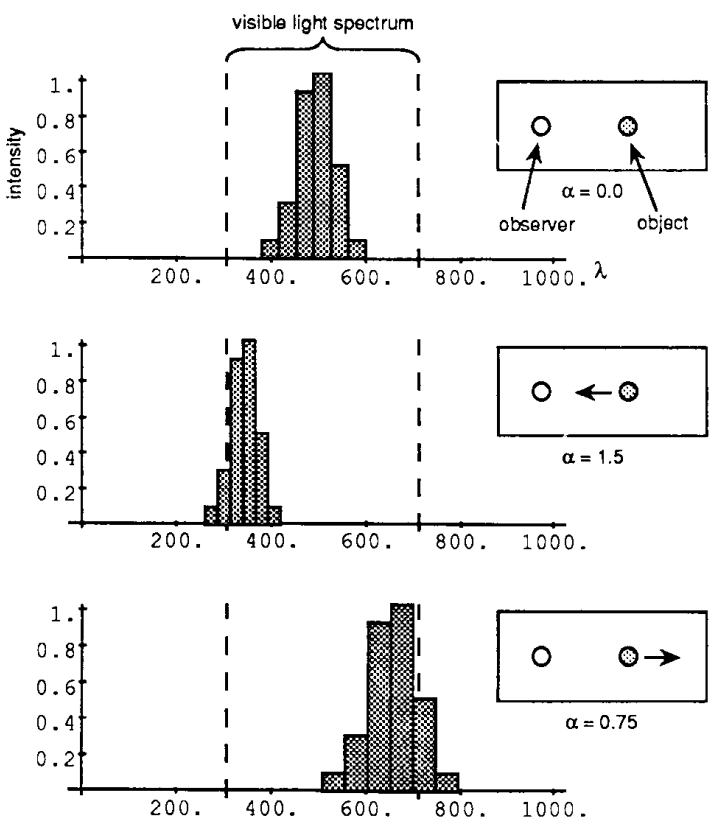

Figure 4: Doppler shift operations represented by spectral lines (a) source intensity (b) after upshift (c) after downshift.

- shading: Two sets of lines were multiplied by their matching components in each shading calculation to form a new line set.

\subsection{High simulation cost}

To satisfy the wide-band representation requirement of section 2.4 , the sample arrays were necessarily large. For example, representing a spectral curve from $50 \mathrm{~nm}$ to 5000 $\mathrm{nm},{ }^{2}$ at the resolution of $5 \mathrm{~nm}$ interval, would result in 991 sample data. To describe object's surface optical characteristics, four curves would then be needed for diffuse reflection, highlight, specular reflection and refraction. This would result in 3964 samples, and twice as many at each node of a shade tree.

Large arrays make Doppler operations numerically expensive. Furthermore, these operations are invoked for every light-surface interaction, and may be performed 1020 times for each screen pixel.

Indeed, our experiments showed that the Doppler operations often incurred over $100 \%$ penalty to the total REST-frame simulation time. In contrast, a REST-frame simulation added only $5 \%$ computation overhead to the non-relativistic ray-tracing when performed without the Doppler effect.

Using spectral sample arrays also incurs high overhead (cost) in memory requirement. The input scene description files also become large and occupy substantial disk space.

\subsection{Other limitations}

Spectral line representation also has poor accuracy and low expandability:

- poor accuracy: As each shift operation changes the interval between lines, a re-sample based on interpolation is employed afterward to produce a new line set that approximates the shifted set but has the proper interval. Each shift thus introduces some error into the line set. The spectral line description of a curve would become increasingly crude after cascaded shifts.

- low expandibility: To expand to wider range or high resolution (e.g. $2.5 \mathrm{~nm}$ ) will worsen the computation and memory costs. The inclusion of thermal or other properties to object specification will have the same effect.

\section{New spline based approach}

This current work builds on the past experiences, and focuses on removing the limitations in the prior investigation and improving the simulation efficiency.

The new approach uses spline functions to represent spectral curves. The Doppler operations of frequency

\footnotetext{
${ }^{2}$ Because most of our data come from spectroscopic work, wavelengths are specified in nanometers.
} 
shift and shading become symbolic manipulations of coefficients, and the re-sampling after each shift is removed. Moreover, the spline approach makes the expansions of wide-band, high resolution description and thermal emission inclusion affordable and accurate.

\subsection{How does the spline representation work}

Cubic splines are an appropriate choice for the representation of surface properties because they are flexible and well understood. For example, a continuous curve representing the diffuse reflection characteristics on an object can be constructed from only a few given data points, each defining the property of the surface for a specific wavelength. Intermediate values can be determined simply by evaluating the curve at a given wavelength. In addition, if greater definition is desired, extra data points may easily be added to the curve.

\subsection{Surface property descriptions}

Once spectral curves are defined as an abstract data type based on splines, they are used to represent the various surface properties required by the REST-frame simulation. In our application, we reason that in the most general case, each object is an emission source in addition to its being reflective and refractive. Surfaces are thus described by five coefficients and splines, plus an index of refraction (IOR). One coefficient and spline each for emission, diffuse reflection, highlight, mirror reflection, and refraction. These are generally enough to describe common, and many uncommon, surfaces or objects. Additional specification can be encompassed similarly.

\subsection{Thermal radiation}

Objects always emit light in the form of thermal radiation. ${ }^{3}$

We include thermal radiation in our Doppler effect simulation for the following reasons:

- The thermal radiation, normally in the IR region, can easily be shifted into the visible range.

- At higher temperatures, the thermal radiation moves into the visible light region, and may become stronger than the object's normal surface characteristics.

- Even at room temperatures, the thermal emission are significant as we may move our observation window to see infrared.

The thermal radiation can be approximated by a blackbody emission curve weighted by the object's emissivity.

\footnotetext{
${ }^{3}$ The converse is not true. That is, all light is not themal. Florescent light, for instance, is based on "cold" radiation.
}

\subsubsection{Blackbody radiation}

A blackbody is a conceptual body with a surface that can absorb all radiation incident upon it (see [2]). In the process of absorption, energy is removed from incident thermal radiation and results in emitting the radiation it absorbed. Since a blackbody absorbs all radiation incident to it, it has zero surface reflectivity.

The spectral distribution of blackbody radiation is defined by the energy emitted per second in radiation of wavelength $\lambda$, and is determined from the surface temperature. Specifically, at temperature $T$ (in Kelvin), the blackbody radiation energy density $E(\lambda)$ is:

$$
E(\lambda)=\frac{3.742 e 2}{\lambda^{5} e^{\left(\frac{1.439 e^{27}}{\lambda T}-1\right)}}
$$

The wavelength range of a blackbody curve $E(\lambda)$ can be loosely characterized by $\lambda_{\max }$ at which $E(\lambda)$ reaches the maximum and by the left and right $1 \%$ wavelengths at which $E(\lambda)$ is $1 \%$ of the maximum. All three wavelengths are uniquely defined by the $T$ as follows:

$$
\begin{aligned}
\lambda_{\max } & =2.898 e 6 / T \\
\lambda_{\text {right } 1 \%} & =6.5 \lambda_{\max } \\
\lambda_{\text {lef } t 1 \%} & =0.33 \lambda_{\max }
\end{aligned}
$$

\subsection{Doppler operations on splines}

The Doppler operations are applied to splines as follows:

- Wavelength shift: The Doppler shifts on the curves are accounted for by "change of variable" - multiplying the independent variable (i.e. wavelength) by the shift factor before the curve is actually evaluated.

- Shading using splines: Integrating two curves is a simple operation - each curve is evaluated at a given wavelength, and the two values are multiplied together. This is applied to every light interaction emission, diffuse, highlight, specular and transmission terms.

\subsection{Final evaluation of pixel color}

After the screen ray has been traced and the shade tree is completed, the tree is passed to another function for evaluation. This function applies the Doppler shift values to the spline coefficients and evaluates the splines in the tree. The final spectrum is converted first into $\mathrm{XYZ}$ and then into an RGB color. Since all of the spectral information is passed into the evaluating function, any region of the EM spectrum can be visualized by changing the evaluation routine. 


\section{Implementation}

In contrast to traditional ray-tracing, the spline-based shade tree must be maintained in memory and evaluated only after it is completely constructed. In order to generate the RGB equivalent of the visible portion of a spectral curve, it must be sampled at a number of points. This is tricky because the curve is the product of all of the curves in the shade tree that describe it, with each curve adjusted for its distortion caused by Doppler shift and attenuation. Ideally, one would like to multiply and adjust the curves as the tree is constructed, and produce a single new spline as a result. In practice, however, multiplying various shifted splines together to produce other splines turns out to be a rather intensive operation. Experiments reveal that it is easier and more efficient to create the entire shade tree first and then traverse it each time one wishes to find its value for a specific frequency of light. ${ }^{4}$

\subsection{Spline representation}

The particular spline used in our implementation is "Cardinal" cubic. The surface characteristics are passed in as wavelengths and intensities from the scene input file. The program then pre-calculates the spline data and stores in structure for later use.

Because each curve is usually shared by a large number of primitives, pointers are used to reference them indirectly. The color and blackbody radiation definitions for each object are represented by separate curves.

\subsection{Blackbody radiation curve}

The spline approximation for the blackbody radiation curve is determined upon a few data points. The data points included the left and right one percent point, the maximum point of the curve, and intermediate points between the maximum and minimum points.

\subsection{Doppler frequency shift}

We apply the inverse of the calculated shift to the spline coefficient, then evaluate the spline at the new wavelength. For each shift operation, we store only shift factor $(\alpha)$ at each node of shade tree. The stored $\alpha$ values are used in the evaluation of the shade tree after it is constructed. The benefit for this scheme is that sometimes we can perform tree depth compression for consecutive shifts of common curves.

\footnotetext{
4 as opposed to creating entirely new splines that represent the evaluation of the entire shade tree, and evaluating them.
}

\subsection{Shading and stack evaluation}

The shading function determines how objects will interact with light; it is expressed as the emission, diffuse, highlight, transmission and reflection terms and splines. For each surface intersection, a structure (or shading node) is created. The calculated coefficients and pointers to the surface and light data are placed in the node. Reflections and transmission are handled by calculating new rays, calling the tracing function recursively, and then storing pointers to the node(s) created. The new node is then returned to the calling function. This creates a "shade tree" which contains all of the coefficients and spline data needed to calculate the final color. Because the shader doesn't have to manipulate the splines directly, or make any assumptions about the data it is handling, a great deal of calculation time is saved.

\subsection{Spline spectrum to RGB color conver- sion}

To preserve the spectral information, the spectral spline is only converted back to RGB once the light reaches the screen or eye. Since the spectrum has been shifted and modified along the path of the ray, and the final spectral color is returned to the eye, only the visible portion of the spectrum is used in the conversion.

\section{Experiments: speed up over spec- tral line based implementation}

Our previous discrete spectral line approach incurred over $100 \%$ overhead in the REST-frame simulation. That is, in each simulation, the Doppler-related functions consumed over $50 \%$ of the total time. Other REST-frame functions (ray-firing, ray origin transformation and direction aberration, and ray-object intersection) amounted to the rest, less than half, of the simulation time. In the new spline code implementation, the Doppler code takes about $2 \%$ of the total simulation time. Using the REST-frame computation time as the base (100\%), the new Doppler code achieves 50 times speed improvement over the old implementation.

\section{Images}

In this section, we show images from our animations that demonstrate the wide-band Doppler effect simulation results.

\subsection{Lattice at 0.99c: Before and after shift}

In this example, a 3D square lattice of finite extent, made of grey bars and red spheres, is viewed at a velocity of $0.99 \mathrm{c}$. 
The lattice is heated up with separate temperatures for red spheres $(300 \mathrm{~K})$ and grey bars $(600 \mathrm{~K})$. Light sources in front of and within the lattice are moving with the lattice. The background is moving with the observer. Different components of relativity are exhibited. Throughout, the severe spatial distortion is visible. Wide-band spectral aspects of relativity are made apparent; the Doppler color shift of the visible spectrum and the shift of the thermal into the visible are seen.

- Plate 1 shows the lattice without Doppler shift treatment and without temperature assignment. Only the spatial distortion is presented.

- Plate 2 shows the lattice with Doppler color shift and with temperature assignment. The thermal emission is shifted into the visible band and appears in glowing orange color. The infrared spectrum shifted into the visible frequency range (blue-shift phenomenon) is apparent. Red spheres at sharpest angle off motion in the foreground are now brown, then eventually turn to yellow and become saturated towards the center or least angle of viewing. Grey bars turn light orange in the sharp angle foreground, then become yellow and reach saturation towards the center of the lattice at lesser angles off motion direction. Therefore, this image demonstrates that apparent color is dependent on angle of observation. The highest shift factor is in the center, at zero angle between direction of velocity and observed direction of light. Lowest shift is at the sharpest viewing angles off motion direction (blackbody curve has just begun to enter or has not yet entered visible frequency range).

\subsection{Array of Spheres}

A 3D array of red spheres is used in this example (see figure 5). Four lights out front are moving with the ar-

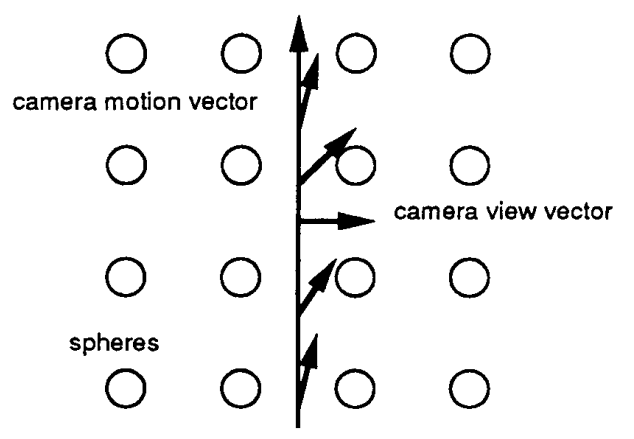

Figure 5: Diagram of camera motion and panning for Plate $3,4,5$, and 6 .

ray, the background color is moving with the observer (not shifted). The camera/observer enters the array which moves at the velocity of $0.25 \mathrm{c}$, and pans from 0 to $75 \mathrm{de}$ grees. The observer views a range of colors based on the variation of direction- dependent shift.

- Plate 3 is a static array of red spheres.

- In Plate 4, the camera/observer enters the sphere array which is moving at $0.25 \mathrm{c}$. At 0 degrees between motion direction and observed light direction, visible color is shifted from red to green (blue-shift). In this spliced image, the lower half shows the sharp increase of light intensity for forward motion caused by the relativistic beaming.

- In Plate 5, the observer looks off the direction of motion at 51.25 degrees. Spheres within the field of vision are seen to have colors across a green, (lime), yellow to orange range.

- Plate 6 is a sharp look off the direction of motion at 75 degrees. The observer views a foreground sphere shifted to orange, and a crossover region with spheres of the original red color. Note that beyond this region, an ultraviolet definition would shift into the visible range, as this would be perceived to be a red-shifted area.

\subsection{Rows of spheres}

In this set of images, several rows of red spheres, with wide-band spectral characteristics, cut the observer's viewing plane (see figure 6 ). The spheres, each with a

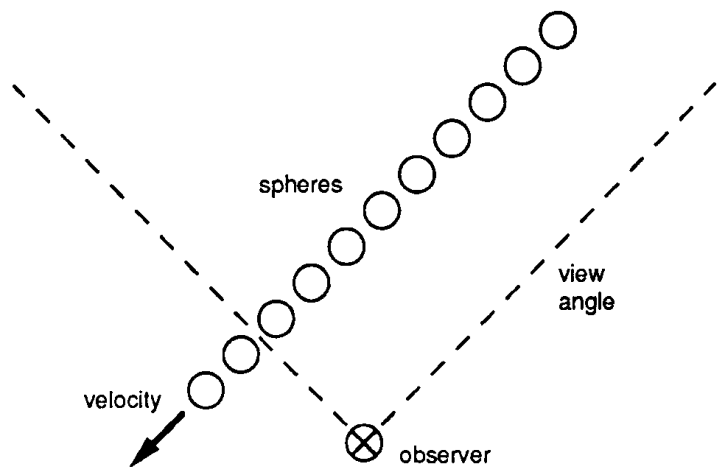

Figure 6: Diagram of imaging configuration for Plate 7 through 12.

velocity of $0.5 \mathrm{c}$, move across the viewer's 90 degree wide field of vision. The viewing angle is 45 degrees with respect to the motion direction of the rows. Light sources are moving with the rows of spheres, the background is moving with the observer. Spheres are seen initially at no velocity (static) and $T=0$. The velocity is increased gradually from $0.0 \mathrm{c}$ to $0.5 \mathrm{c}$, however, the time parameter 
does not change; this is not acceleration. This is analogous to stop-frame character animation. At the fixed velocity of $0.5 \mathrm{c}$, the temperatures for the rows of spheres are incrementally increased from $0 \mathrm{~K}$ to approximately $20000 \mathrm{~K}$. The thermal radiation moves into the visible light region, becoming stronger than the object's normal surface characteristics. Note the loss of dimensional quality of the spheres as thermal shifts into the visible. Due to relativistic distortion, the viewing angle appears wider, sphere rows appear to shear and become more densely packed at the $0.5 \mathrm{c}$ velocity. (However, elliptical shapes in near extreme areas are caused by wide angle distortion due to projection transformation.) At higher temperatures, thermal shift visibility diminishes, as defined blackbody curves have reduced intensity in the visible range, and natural color (based on surface reflection/definition) becomes apparent again. The curves and their superimposition describe characteristics of a pixel at center screen.

- Plate 7 has rows of red spheres at zero velocity, with all spheres at the temperature $T=0$.

- Plate 8 shows the visible color shift of spheres at the velocity of $0.5 \mathrm{c}$ with $T=0 \mathrm{~K}$. Note the severe blueshift, including complete shift out of color. Evident is the crossover point return to original color in the far left region of the field of vision.

- Plate 9 has spheres at velocity of $0.5 \mathrm{c}$ and with $T=1663 K$ (the melting temperature of titanium). The invisible infrared frequencies have shifted into the visible spectra. Note the confluence of original color with infrared frequencies that get shifted into the visible spectra.

- Plate 10 shows spheres at velocity of $0.5 \mathrm{c}$ and $T=$ $2000 \mathrm{~K}$. The observer sees the crossover point region; with original red color in the far left region from the center line of viewing. Thermal shift color (e.g. yellow) is seen to spread at more remote, off angle regions.

- Plate 11 exhibits an extreme thermal shift at velocity of $0.5 c$, and temperature $T=4000 \mathrm{~K}$. There is a high intensity saturated wash-out of most prior visible color.

- Plate 12 shows that the color shift based on surface reflection and definition makes a visible contribution at the higher temperature of $10000 \mathrm{~K}$, as the predominant blackbody spectral curve moves out of the visible spectrum.

\section{Conclusion}

The REST-frame algorithm has a generalized capability to describe wide-band Doppler shift effects. This is accomplished through the manipulation of spline functions which represent spectral curves of surface properties and light composition.

Our simulations of relativistic effects may provide insight into the detailed workings of the universe, and may provide a visualization paradigm in which researchers can experience and interpret phenomena such as those of astrophysics.

\section{Acknowledgments}

We thank our reviewers for their valuable comments. We are grateful to Joel Welling and Anjana Kar of the Pittsburgh Supercomputing Center for their constant assistance. We also thank Jim Kocher and Ken Mohnkern of the Robotics Institute, Carnegie Mellon for their help in preparing our animations. Finally we appreciate the comments from Quan Le, Kathryn Porsche, Wendy Plesniak, and Lily Dong and David Thuel of the Imaging Systems Laboratory.

\section{References}

[1] Robert Cook. Shade trees. Computer Graphics (SIGGRAPH), pages 223-231, July 1984.

[2] Richard P. Feynman, Robert B. Leighton, and Matthew Sands. The Feynman lectures on physics. Addison-Wesley, 1963.

[3] Ping-Kang Hsiung and Robert H. P. Dunn. Visualizing relativistic effects in spacetime. In Proceedings of the Supercomputing '89 Conference, Nov. 13-17, 1989.

[4] Ping-Kang Hsiung, Robert H. Thibadeau, Christopher B. Cox, and Robert H. P. Dunn. Doppler color shift in relativistic image synthesis. International Conference on Information Technology (Tokyo, Japan), October 1990

[5] Ping-Kang Hsiung, Robert H. Thibadeau, and Michael Wu. T-Buffer: Fast visualization of relativistic effects in spacetime. In 1990 Symposium on Interactive $3 D$ Graphics, March 18-21, 1990.

[6] James T. Kajiya. The rendering equation. Computer Graphics (SIGGRAPH), page 143, July 1986.

[7] C. Møller. The Theory of Relativity. Clarendon Press, Oxford, 1972.

[8] V.F. Weisskopf. The visual appearance of rapidly moving bodies (section). Physics Today, 13(9):24, 1960. 


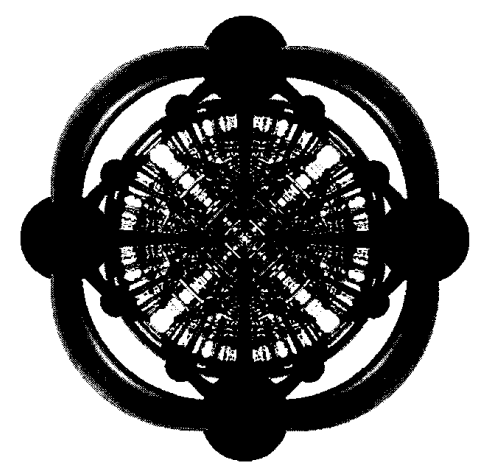

Plate 1: 3D square lattice at $0.99 \mathrm{c}$, without Doppler shift or temperature. (Color Plate 33, page 465)

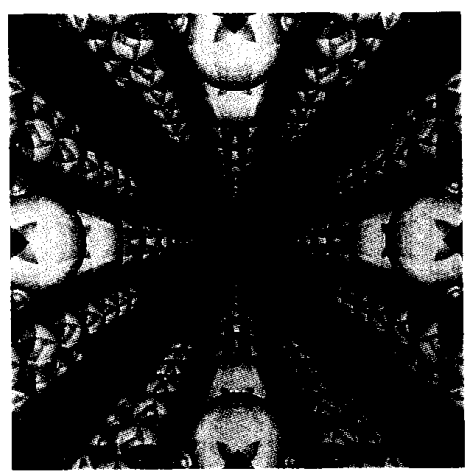

Plate 3: Static array of red spheres. (Color Plate 35, page 465)
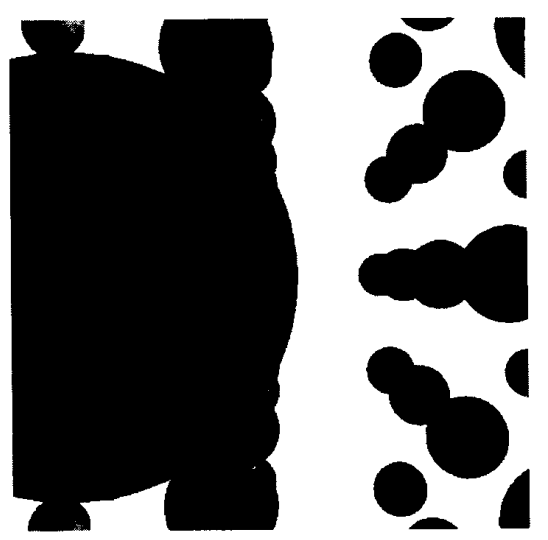

Plate 5: Sphere array viewed at 51.25 degrees off direction of motion. (Color Plate 37, page 466)

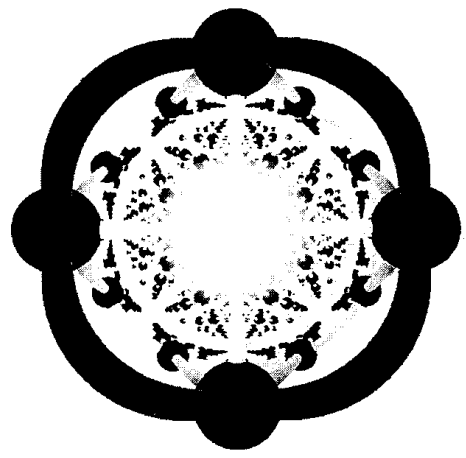

Plate 2: 3D Square lattice at $0.99 \mathrm{c}$, with Doppler shift. (Color Plate 34, page 465)

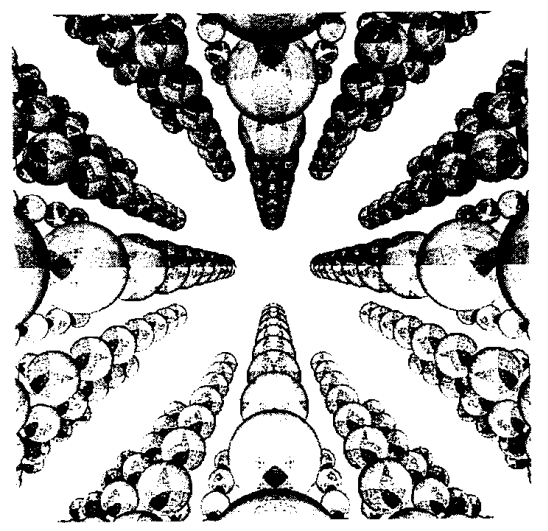

Plate 4: Sphere array at $0.25 \mathrm{c}$ towards observer (blue-shifted). (Color Plate 36, page 465)

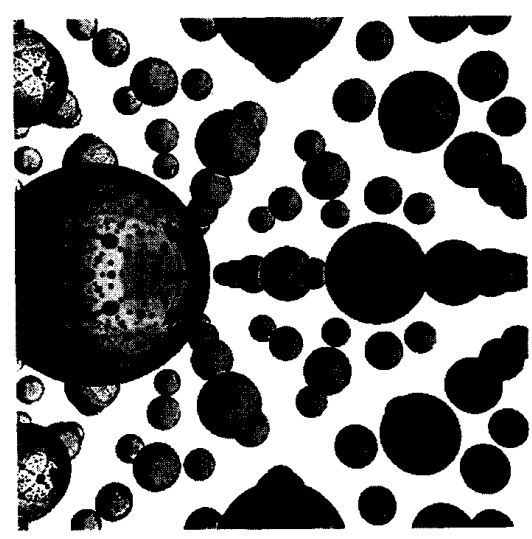

Plate 6: Sphere array viewed at 75 degrees off direction of motion. (Color Plato 38, pago 468) 


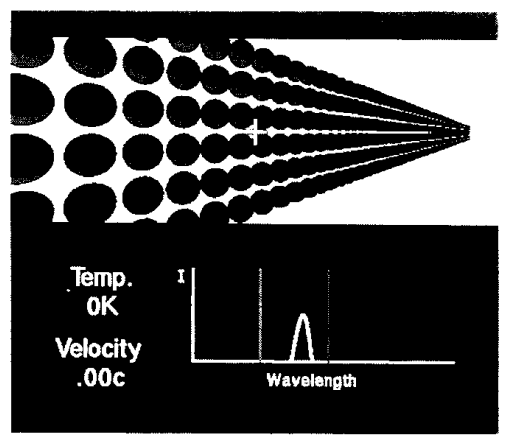

Plate 7: Static rows of red spheres with spectral emission plot. (Color Plate 39, page 466)

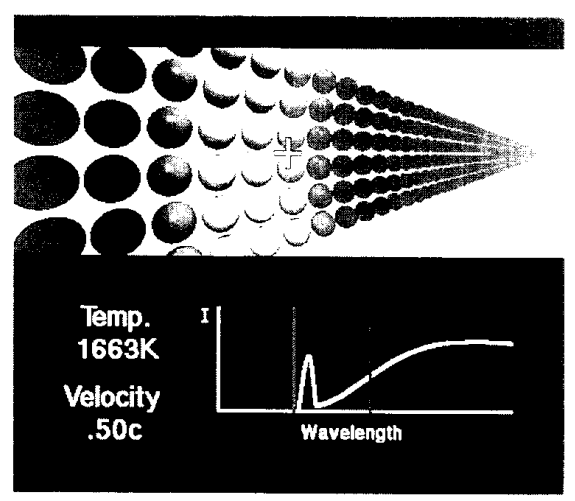

Plate 9: Sphere rows at $0.50 \mathrm{c}$ and temperature $\mathrm{T}=1663 \mathrm{~K}$. (Color Plate 41, page 466)

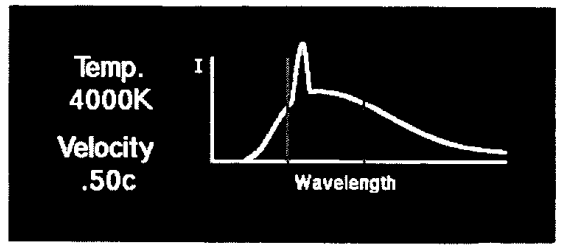

Plate 11: Sphere rows at $0.50 \mathrm{c}$ and temperatur. $\mathrm{T}=4000 \mathrm{~K}$. (Color Plate 43, page 467)

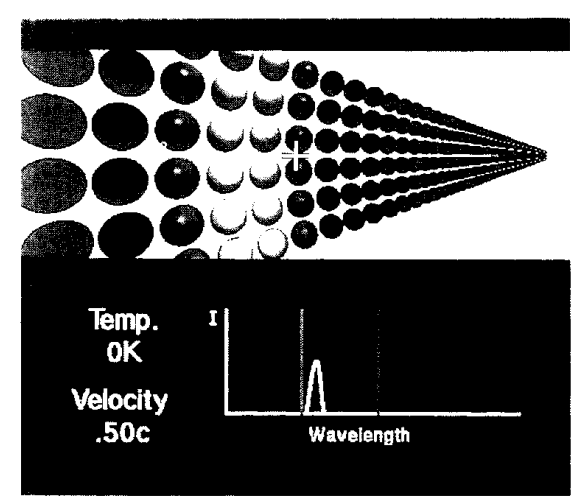

Plate 8: Sphere rows at $0.50 \mathrm{c}$ and temperature $T=0$. (Color Plate 40, page 466)

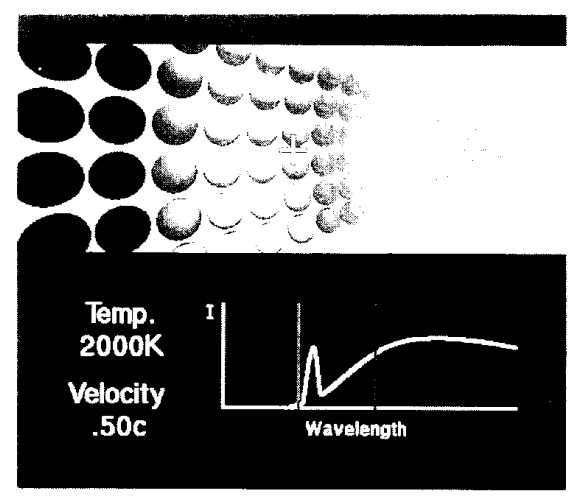

Plate 10: Sphere rows at $0.50 \mathrm{c}$ and temperature $\mathrm{T}=2(100 \mathrm{~K}$. (Color Plate 42, page 466)
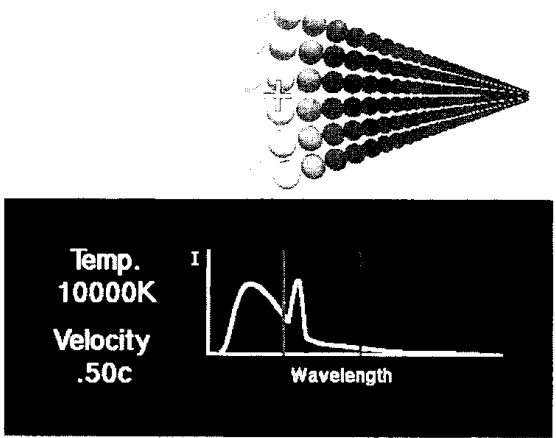

Plate 12: Sphere rows at $0.50 \mathrm{c}$ and temperature $r=10000 \mathrm{~K}$. (Color Plate 44, page 467) 